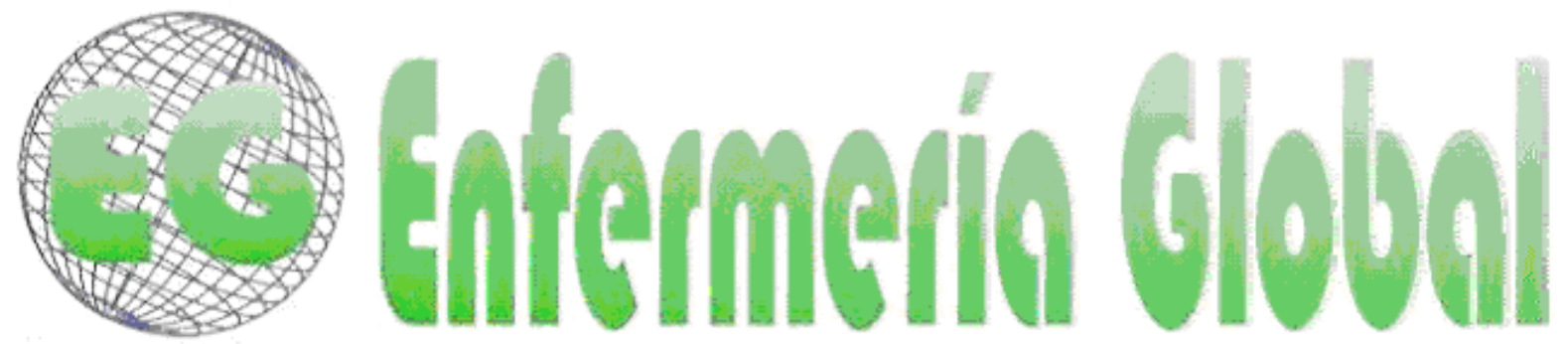

\title{
Prevalencia de los factores de riesgo cardiovascular en trabajadores de los sectores laborales secundario y terciario
}

Prevalence of cardiovascular risk factors in workers in secondary and tertiary sectors of employment

\author{
*Martínez Abadía, B. *Arbués, ER. \\ *Universidad San Jorge. Zaragoza. E-mail: martinezabadia@hotmail.com \\ Palabras clave: enfermedad cardiovascular; factores de riesgo cardiovascular; riesgo cardiovascular; \\ promoción de la salud. \\ Keywords: cardiovascular disease; cardiovascular risk factors; cardiovascular risk; health promotion.
}

\section{RESUMEN}

Los reconocimientos médicos de los trabajadores constituyen una oportunidad de detección precoz de factores de riesgo cardiovascular. Por ello se planteó conocer la prevalencia de los factores de riesgo cardiovascular en los trabajadores de dos sectores laborales, estimar el riesgo cardiovascular individual y evaluar las diferencias entre los sectores.

Se diseñó un estudio descriptivo retrospectivo de tipo transversal. La población estudiada estaba formada por 15.771 trabajadores que han acudido a reconocimiento médico laboral durante el año 2009. Se estudiaron los siguientes factores de riesgo cardiovascular: edad, sexo, diabetes, hipertensión, tabaquismo, obesidad, actividad física, colesterol, HDL y riesgo cardiovascular.

El $44,3 \%$ de los trabajadores pertenecen al sector servicios y el $55,7 \%$ al sector industria. La edad media fue de 46,27 años. El $73,1 \%$ son hombres y el $26,9 \%$ mujeres. Las prevalencias estimadas fueron: diabetes 2,9\%; hipertensión 3,2\%; tabaquismo $43,7 \%$; sobrepeso $38,4 \%$ y obesidad el $19,4 \%$; sedentarismo 56,3\%; dislipemias (colesterol alterado el 39,5\%, elevado el $19,7 \%$ y el HDL bajo < de $40 \mathrm{mg} / \mathrm{dl}$ el $8,3 \%$ ); y el riesgo cardiovascular (SCORE) es bajo de $0,62 \%$ en el sector terciario y $1,03 \%$ en el sector secundario. Se han encontrado diferencias significativas entre los sectores: en el sedentarismo, el colesterol HDL y el riesgo cardiovascular, el sector secundario prevalece sobre el sector terciario.

\section{ABSTRACT}

The medical examination of workers is an opportunity for the early detection of factors of cardiovascular risk.

We designed a retrospective transverse study. The studied population consisted of 15,771 workers who attended medical examinations in 2009. We studied the following cardiovascular risk factors: age, sex, 
diabetes, hypertension, smoking, obesity, physical activity, cholesterol, HDL-cholesterol and cardiovascular risk.

$44.3 \%$ of workers belong to the service sector and $55.7 \%$ to the industrial sector. The mean age was 46.27 years old. The estimated prevalence for diabetes was $2.9 \%, 3,2 \%$ for hypertension, $43,7 \%$ for smoking, $38.4 \%$ for being overweight, $19,4 \%$ for obesity, $56.3 \%$ for sedentary lifestyle, $39,5 \%$ for altered cholesterol and cardiovascular risk (SCORE) at $0.62 \%$ in the service sector and at $1,03 \%$ in the industrial sector.

We found significant differences between sectors about physical inactivity, HDL and cardiovascular risk.

\section{INTRODUCCIÓN}

Para la Organización Mundial de la Salud (OMS) (1), las enfermedades cardiovasculares (ECV) son la principal causa de muerte en todo el mundo. España no constituye ninguna excepción, según datos recopilados por el Instituto Nacional de Estadística (INE) ${ }^{(2,3)}$, las ECV se mantuvieron como primera causa de muerte en el conjunto del año 2008, representando un 31,7\%.

Los factores de riesgo cardiovascular (FRCV) ${ }^{(4-6)}$ son unas características biológicas o conductas que, en aquellos individuos que las presentan, aumentan la probabilidad de padecer una ECV o morir por esa causa.

El riesgo cardiovascular $(\mathrm{RCV}){ }^{(7-8)}$ se define como la probabilidad que tiene un sujeto de presentar una ECV, cardiopatía isquémica o enfermedad cerebrovascular, en un período de tiempo determinado, generalmente 10 años.

Con el estado del conocimiento actual sobre FRCV, no podemos saber con certeza absoluta si un paciente va a sufrir o no un evento cardiovascular en su vida. Por esto tenemos que utilizar un modelo probabilístico y efectuar una valoración global del riesgo mediante modelos multifactoriales, que predicen el riesgo individual de forma más exacta y permiten una utilización más eficiente de la medición disponible. La información a los pacientes de su RCV puede ayudarles a modificar dichos factores de riesgo y por tanto a prevenir las ECV.

Teniendo en cuenta que una de las actividades prioritarias de la Medicina y Enfermería del Trabajo es la prevención y la promoción de la salud, no se deben escapar las ECV en su conjunto.

En el artículo 38 del Reglamento de los Servicios de Prevención Españoles ${ }^{(9)}$, de acuerdo con lo establecido en el artículo 10 de la Ley 31/1995, de Prevención de Riesgos Laborales, y artículo 21 de la ley 14/1986, General de Sanidad, el servicio de prevención colaborará en las campañas sanitarias y epidemiológicas organizadas por las Administraciones públicas competentes en materia sanitaria.

En la actualidad, la mejor manera de predecir la aparición de $\operatorname{ECV}^{(7-8)}$ es la detección de factores de riesgo que se sabe que están asociados a una mayor predisposición para el desarrollo de estas enfermedades.

Los reconocimientos médicos que se hacen en los centros de trabajo, son la oportunidad perfecta para llevar a cabo una prevención de la ECV en la población trabajadora. Puesto que nos ayudan a identificar a los trabajadores con riesgo cardiovascular elevado, sobre todo, en edades en las que no se suele acudir al 
médico por no tener problemas de salud aparentes. Gracias a la detección de los FRCV, permite la puesta en marcha de las medidas preventivas de un modo precoz. A este respecto, hay consenso sobre la necesidad de iniciar la prevención de la arteriosclerosis en etapas tempranas de la vida, especialmente en lo que se refiere al fomento de hábitos de vida más saludables.

Los distintos sectores productivos en el ámbito laboral ${ }^{(10)}$ : primario, secundario o industrial y terciario o servicios, condicionan un tipo de actividad distinto que pueden determinar diferencias en la prevalencia de los $\mathrm{FRCV}$, lo que se pretende aclarar con este estudio.

\section{MATERIAL Y MÉTODOS}

Se diseñó un estudio descriptivo retrospectivo de tipo transversal. La población laboral estudiada estaba formada por 15.771 trabajadores que han acudido a reconocimiento médico laboral durante el año 2009 en los centros médicos de la Sociedad de Prevención de Maz de la capital de Zaragoza, pertenecientes a dos sectores laborales: sector productivo secundario y sector productivo terciario. En la población, de 15.771 sujetos, 8.774 proceden del sector secundario (el 55'63\%) y 6.997 proceden del sector terciario (el restante 44 '37\%).

La recogida de la información se realizó entre enero y marzo de 2010 de la historia clínico-laboral de los trabajadores que pasaron reconocimiento médico durante el año 2009.

Se preparó una ficha de recogida de datos que recopilaba: 1. Datos demográficos: edad (35-65 años), sexo, sector productivo al que pertenecía. 2. Antecedentes y hábitos de salud: Diabetes, Hipertensión, Hábito de tabaquismo y actividad física. 3. Medidas antropométricas (peso y talla) y toma de la presión arterial. 4. Datos analíticos: Colesterol y HDL-colesterol.

Para calcular el riesgo cardiovascular se ha utilizado la tabla de SCORE ${ }^{(11)}$, estima el riesgo a los 10 años de enfermedad cardiovascular mortal. El cálculo se basa en las variables edad, sexo, colesterol total, colesterol ligado a proteínas de alta densidad (HDL), tensión arterial (Sistólica y Diastólica) y consumo de tabaco. La diabetes se considera de alto riesgo y por tanto en esta tabla no contempla su cálculo.

Clasificación del riesgo de sufrir una enfermedad cardiovascular mortal a los 10 años: 1.Muy bajo, con riesgo inferior al 1\%; 2. Bajo, con riesgo del 1\%; 3. Ligero, con riesgo del $2 \%$; 4. Moderado, con riesgo entre el $3 \%$ y el $4 \% ; 5$. Alto, con riesgo del $5 \% 0$ superior.

Los datos han sido analizados mediante el paquete estadístico SPSS versión 15. Los resultados de las variables categóricas 0 cualitativas se presentan como porcentajes(\%) con intervalos de confianza (IC) del 95\%. En el análisis de relación entre estas variables se ha utilizado la prueba Chi-Cuadrado. En las variables cuantitativas los resultados se presentan con la media con IC del $95 \%$. En la comparación de estas variables en los grupos de las variables categóricas se ha utilizado la prueba de Mann-Whitney. El nivel de significación elegido ha sido 0,05. El estudio fue aprobado por el Comité de Ética y se mantuvo la confidencialidad establecida por la vigente Ley Orgánica de Protección de Datos. 


\section{RESULTADOS}

En la población laboral estudiada se obtiene la siguiente distribución de frecuencias: el $44,3 \%$ de los trabajadores trabajan en el sector terciario; mientras que el restante $55,7 \%$ lo hace en el sector secundario.

Se observa que la media de edad de los trabajadores es de 46'27 años. La media de la edad entre los trabajadores del sector secundario (45'87 años) es ligeramente inferior a la del sector terciario (46’78 años); sin apreciarse diferencias significativas.

Se comprueba que la proporción de hombres $(73,1 \%)$ que acuden a reconocimiento médico laboral casi triplica a la de mujeres $\left(26^{\prime} 9 \%\right)$. Así como en el sector terciario los trabajadores que acuden a la consulta se distribuyen casi por igual entre ambos sexos (con un $53^{\prime} 6 \%$ de hombres y un $46^{\prime} 4 \%$ ), en el sector secundario son hombres en una amplia mayoría (el 88 '5\%).

La proporción de trabajadores que afirman tener diabetes es del 2'9\%. En el sector terciario representa el 2'4\%; mientras que en el sector secundario es sólo ligeramente superior, el 3'3\%, sin apreciarse diferencias significativas entre los sectores.

La prevalencia de la hipertensión en la población laboral estudiada es de 3,2\%. En el sector terciario es del 1'8\%; mientras que en el sector secundario es ligeramente superior, el $43 \%$, sin diferencias significativas en los porcentajes de hipertensos en ambos sectores. Se les tomó la presión arterial en el reconocimiento médico a los trabajadores y se obtuvieron los siguientes resultados: el 23,2\% tuvo presión arterial "normal-alta", el $18,4 \%$ cifras superiores o iguales a $140 / 90 \mathrm{mmHg}$.

El $47,1 \%$ de los trabajadores ha manifestado que son fumadores en la actualidad, siendo mayor el porcentaje en el sector secundario pero sin apreciarse diferencias significativas entre los sectores.

El $38,4 \%$ posee "sobrepeso" y el $19,4 \%$ "obesidad". En general, a nivel descriptivo, parece que se dan mayores niveles de obesidad en el sector secundario, pero las diferencias no son lo suficientemente elevadas como para que resulten significativas.

Se comprueba que la mayoría de los trabajadores (el 56,3\%) han manifestado que llevan una vida sedentaria en relación con la actividad física que desarrollan. Hay considerables diferencias entre los sectores, mientras que en el sector secundario el porcentaje de trabajadores que afirma llevar una vida sedentaria es del $64,1 \%$, en el sector terciario es bastante menor $46,4 \%$, apreciándose diferencias significativas entre los sectores (Figura 1). 
Figura 1. Relación actividad física por sectores de producción.

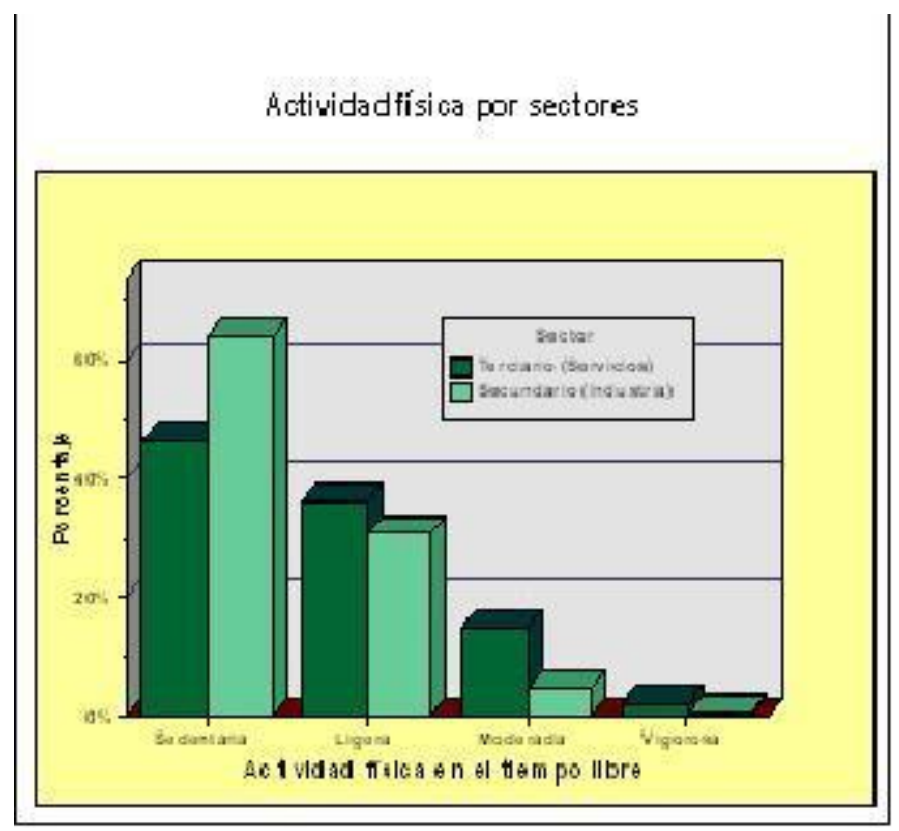

En los resultados obtenidos son muy similares los porcentajes de colesterol total de los trabajadores de ambos sectores: el 19,7\% posee un colesterol total "elevado" y el $39,5 \%$ lo tienen en "límite alto". Se determinaron las cifras de HDL con una prevalencia del $10,7 \%$. Los niveles de cHDL se elevaron significativamente según el tipo de sector, en el sector secundario el cHDL bajo fue del 13,9\% frente al sector terciario de un $6,6 \%$, apreciándose diferencias significativas entre los sectores.

Figura 2. Relación colesterol HDL por sectores de producción.

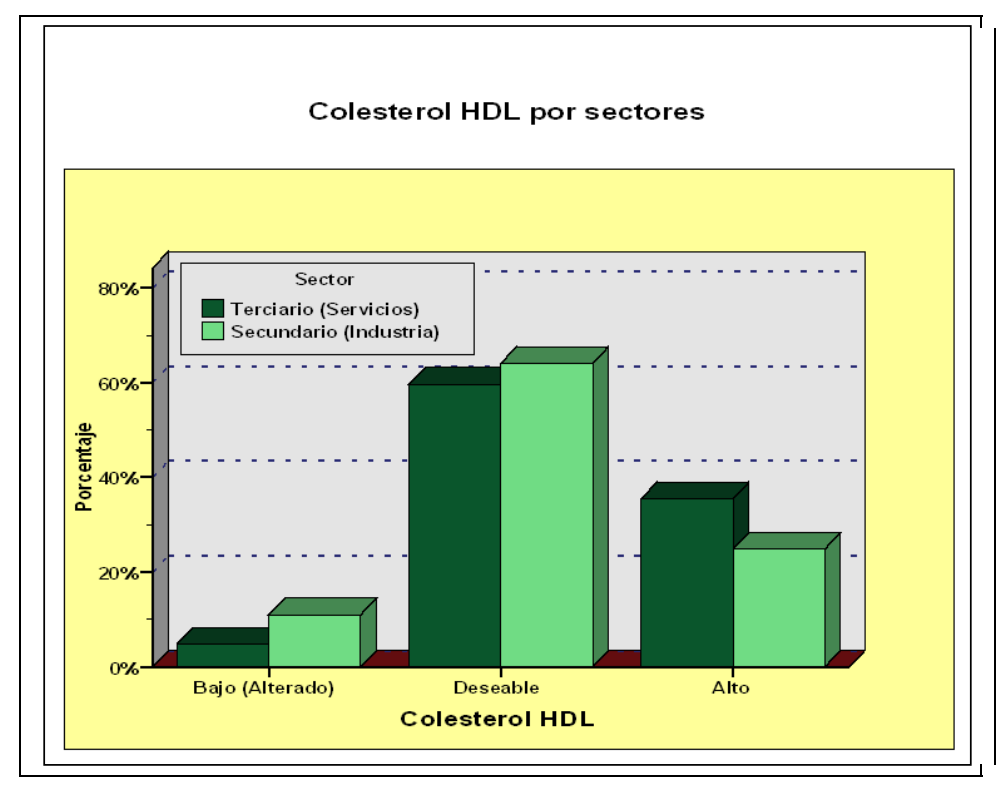

El riesgo cardiovascular es mayoritariamente "muy bajo"; ya que en ese nivel se encuentra el $55^{\prime} 5 \%$ de los trabajadores. Otro amplio $26^{\prime} 4 \%$ de los pacientes presenta un riesgo cardiovascular "bajo", "ligero" el 8\%, "moderado" el 6,7\% y riesgo "alto" el 
$3,2 \%$. En el sector secundario se han obtenido los niveles más altos de riesgo cardiovascular, encontrándose diferencias significativas con el sector terciario.

Figura 3. Relación riesgo cardiovascular por sectores de producción.

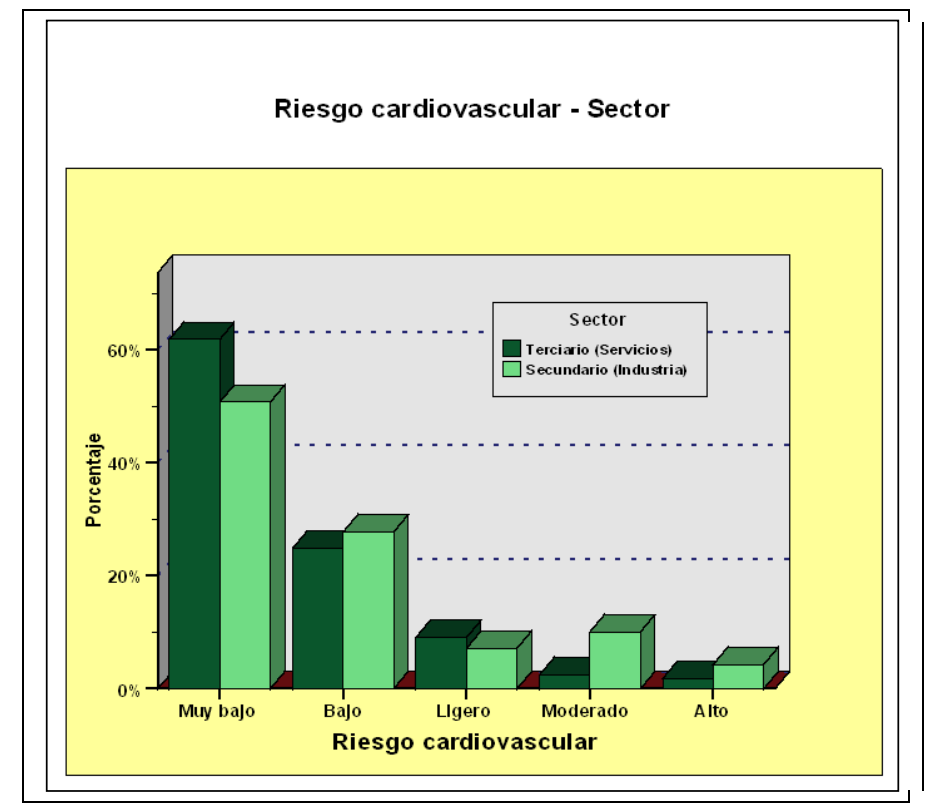

\section{DISCUSIÓN}

La muestra estudiada representa, obviamente, a una población seleccionada de la población laboral de Zaragoza perteneciente a la Sociedad de Prevención de Maz. Los resultados ofrecen una visión precisa de un problema frecuente en un grupo de población, mayoritariamente joven, y con posibilidades de intervención.

Es difícil comparar las prevalencias de los FRCV debido a las diferencias en los métodos utilizados, los grupos de edad estudiados, la estandarización de la población, los factores estudiados y los límites considerados en cada factor como patológicos.

Las respuestas de los trabajadores a las preguntas relativas a la actividad física y al consumo de tabaco, al no poder ser verificadas, presentan el margen de error que entraña toda respuesta subjetiva, que puede estar condicionada por factores que no están al alcance.

La proporción de trabajadores que afirman tener diabetes resulta ser inferior a la prevalencia de diabetes en España ${ }^{(12-16)}$ que gira en torno a un 6,5\% en edades similares a las del estudio y en el estudio Manresa ${ }^{(17)}$ (en población laboral) la prevalencia de diabetes reportó un 7,9\%. Pero a la vez el porcentaje de diabetes es similar a otros estudios en población laboral, en el estudio realizado por lbermutuamur ${ }^{(18)}$ el 2,4\%, el 2,3\% en el estudio realizado por Castan FJ y Gutiérrez $M^{(19)}$.

La prevalencia de hipertensión en el estudio es considerablemente inferior respecto a otros estudios, un reciente metaánalisis realizado por Mediano et al ${ }^{(20)}$ arroja una cifra del $34 \%$, el $13,3 \%$ en el estudio realizado por Castan FJ y Gutiérrez $M^{(19)}$, en el estudio realizado por Ibermutuamur ${ }^{(9)}$ el $6,2 \%$. También se les tomó la presión arterial $^{(21,22)}$ en el reconocimiento médico y los resultados difieren puesto que el $23,2 \%$ 
presentaban T/A normal alta y el 18,4\% HTA. Merece la pena destacar las cifras tan dispares objetivadas al contrastar la prevalencia de la HTA referida en los antecedentes de enfermedad que fue del 3,2\%, y la objetivada según cifras tensiónales de cada trabajador, de acuerdo con la OMS/Sociedad Internacional de Hipertensión y Joint National Committee on Prevention for High Blood Pressure. Este contraste es debido a dos circunstancias fundamentales: 1) los trabajadores que no están trabajando en empresas con servicio médico o tutelados de alguna otra manera, no han podido ser etiquetados de hipertensos; 2) Los criterios de clasificación de HTA, al ser más restrictivos, hacen que entren a formar parte del grupo de tensión arterial normal alta, que en otras circunstancias no hubieran sido considerados como tales.

La prevalencia de tabaquismo en el estudio (46,7\%) es superior a los datos más recientes en la población general de España que muestran que en el año 2006 la prevalencia de consumo de cigarrillos fue de $26,44 \%$, en el estudio Corsaib ${ }^{(23)}$ fue del $27 \%$, pero similar a la publicada en el estudio realizado por lbermutuamur ${ }^{(18)}$ que fue de un 49,3\%, el 51,3\% en el estudio realizado por Castan FJ y Gutiérrez $M^{(19) \text {. }}$

Los resultados de obesidad obtenidos son similares respecto a la población general española en 2006 (15,56\%) (16), 15,5\% en el estudio realizado por lbermutuamur ${ }^{(18)}$, $15,3 \%$ en el estudio realizado por Castan FJ y Gutiérrez $\mathrm{M}^{(19)}$. La prevalencia de obesidad en el estudio fue considerablemente inferior al estudio Corsaib ${ }^{(23)}$ que fue del $27 \%$ y un sobrepeso del $40,1 \%$.

En el estudio no se puede hablar realmente de sedentarismo, ya que se ha recogido la percepción de los trabajadores sin comprobarlo a través de la medición del gasto calórico, pero permite comparar la declaración del ejercicio realizado fuera del trabajo. La mayoría de los trabajadores (el 56,3\%) han manifestado que llevan una vida sedentaria. En la encuesta nacional de salud del año 2006 en España ${ }^{(24)}$ se declaró sedentaria el $39,44 \%$ de la población, un $44,35 \%$ en el estudio Corsaib ${ }^{(23)}$, una proporción muy inferior a la de nuestro estudio.

La prevalencia de los factores de riesgo lipídicos es elevada en el estudio, a la hora de comparar con otros estudios ${ }^{(25)}$ es muy complicado debido a los límites considerados en el factor como patológico. En el estudio realizado por lbermutuamur (18) el $15 \%$ de la población presentó cifras elevadas de colesterol, en el estudio Corsaib ${ }^{(23)}$ el $24,3 \%$ y el $30 \%$ en el estudio realizado por Castan FJ y Gutiérrez M (19).

Se determinaron las cifras de HDL con una prevalencia del 10,7\%. Difieren los resultados respecto al estudio realizado por lbermutuamur ${ }^{(18)}$ ya que la prevalencia de cHDL bajo $<40 \mathrm{mg} / \mathrm{dl}$ fue de $26 \%$ y similar a los datos encontrados en el estudio realizado por el Grupo de trabajo del Instituto Nacional de Seguridad e Higiene del Trabajo en población laboral ${ }^{(26)}$ que fue de $7,2 \%$.

Al igual que en el estudio realizado por Espina Álvarez y Cols. ${ }^{(27)}$ en dos poblaciones laborales, se comprueba que en relación con el puesto de trabajo que desempeñan, los trabajadores de la construcción pertenecientes al sector secundario tiene un riesgo coronario mayor que los trabajadores ejecutivos pertenecientes al sector terciario (servicios). 


\section{CONCLUSIONES}

En la población laboral estudiada se ha encontrado una alta prevalencia de los FRCV.

En base a los resultados del estudio, el sector secundario (industria) presenta una mayor prevalencia de FRCV respecto del sector terciario (servicios), a pesar de que sólo se aprecien diferencias significativas en la actividad física, en el colesterol HDL y $\mathrm{RCV}$ individual.

El hallazgo de una mayor prevalencia de FRCV en el sector secundario puede facilitar el establecimiento de prioridades en la prevención cardiovascular. Esta predicción debe mover a los profesionales sanitarios, especialistas en salud laboral, a implementar programas de intervención, a través de los servicios médicos o de prevención, en las empresas. Programas que deben tender a disminuir los principales factores de riesgo del tema que nos ocupa y especialmente a reducir las cifras de colesterol y de presión arterial, así como de otra parte, a combatir los hábitos de fumar cigarrillos, fomentar la actividad física y hábitos dietéticos saludables.

\section{BIBLIOGRAFÍA}

1. Organización Mundial de la Salud [sede Web]. 2008. [acceso 10 de febrero de 2009] Enfermedades cardiovasculares. Disponible en:

http://www.who.int/topics/cardiovascular diseases/es/

2. Instituto Nacional de Estadística [sede Web]. España: INE; 2008 [acceso 10 de febrero de 2009]. Defunciones según causa de muerte 2008. Defunciones por causas (lista detallada/reducida), sexo y edad. Disponible en:

http://www.ine.es/jaxi/tabla.do

3. Instituto Nacional de Estadística [sede Web]. España: INE; 2008 [acceso 10 de febrero de 2009]. Defunciones según la causa de muerte 2008. Aragón. Defunciones por provincia de residencia, causas (lista reducida), sexo y edad. Disponible en:

http://www.ine.es/jaxi/tabla.do

4. Alonso Moreno FJ, Casado Pérez P, Díaz- Maroto Muñoz JL, División Garrote JA, Lou Arnal S, Llisterri Caro JL, et al. Área Cardiovascular: Factores de Riesgo Cardiovascular [Internet]. España: Semergen DoC; 2005 [acceso 14 de febrero de 2009]. Disponible en:

http://www.semergen.es/semergen2/microsites/semergendoc/textocardio.html

5. Brotons C, Royo-Bordonada MA, Álvarez Sala L, Armario P, Artigao, Conthe, et al; Comité Español Interdisciplinario para la Prevención Cardiovascular (CEIPC). Adaptación Española de la guía europea de prevención cardiovascular. Rev Esp Salud Pública 2004; 78:435-438.

6. Banegas Banegas JR, Donado Campos JM, Rodríguez Artalejo F, Villar Álvarez F. Las enfermedades cardiovasculares y sus factores de riesgo En España: hechos y cifras. España: Informe SEA 2007; 2007.

7. Armario P, Banegas JR, Campo C, De la Sierra A, Gorostidi M, Hernández R. Guía Española de Hipertensión Arterial 2005 [Internet]. España: Sociedad Española de Hipertensión-Liga Española para la lucha contra la Hipertensión Arterial (SEHLELHA); 2005 [acceso 14 de febrero de 2009]. Disponible en:

http://www.seh-lelha.org/guiahta05.htm

8. Mancia G, de Backer G, DominiczaK A, Cifkova R, Fagard R, Germano G et al. Guía de 2007 para el manejo de la hipertensión arterial [Internet]. Grupo de trabajo para el manejo de la hipertensión arterial de la European Society of Hipertensión 
(ESH) y la European Society Cardiology (ESC); 2007 [acceso 14 de febrero de 2009]. Disponible en:

http://www.seh-lelha.org/pdf/ESHESC2007.pdf

9. Ministerio de Trabajo e Inmigración [Internet]. Madrid: Ministerio de Trabajo y Asuntos Sociales; 1998 [acceso 3 de marzo de 2009]. Reglamento de los Servicios de Prevención y modificación posterior. Disponible en:

$\mu$ http://www.mtas.es/insht/legislation/RD/rd39.htm

10. Instituto Nacional de Estadística [sede Web]. España: INE; 2007 [acceso 3 de marzo de 2009]. Clasificación Nacional de Actividades Económicas. Disponible en:

http://www.ine.es/jaxi/menu.do?type=pcaxis\&path=\%2Ft40\%2Fclasrev\%2F\&file=ineba se\&L=

11. Conroy RM, Pyorala K, Fitzgerald AP, Sans S, Menotti A, De Backer G, et al. Estimation of ten-year risk of fatal cardiovascular disease in Europe: the SCORE project. Eur Heart J [revista en Internet] 2003 [acceso 22 de febrero de 2009]; 24:9871003. Disponible en:

http://www.escardio.org/NR/rdonlyres/E5DD427D-50E2-4F1F-B287C9F24242C29A/0/guidelines SCORE FT 2003.pdf

12. Ministerio de Sanidad y Consumo. Encuesta Nacional de Salud 2006. Estilos de vida: Ejercicio físico en el tiempo libre población adulta [Internet]. España: INE (Instituto Nacional de Estadística); 2006 [acceso 20 de febrero de 2009]. Disponible en:

http://www.msc.es/estadEstudios/estadisticas/encuestaNacional/encuestaNac2006/Es tilosVidaPorcentaje.pdf

13. Regidor E, Gutiérrez-Fisac JL. La salud de la población española en el contexto europeo y del Sistema Nacional de Salud. Indicadores de salud. [monografía en Internet] Madrid: Ministerio de Sanidad y Consumo; 2005 [acceso 18 de febrero de 2009]. Disponible en:

http://www.msc.es/estadEstudios/estadisticas/inforRecopilaciones/tabla/Indicadores5. pdf

14. Ascaso JF, Aguillo E, Araujo D, Becerra A, Calvo FL, et al. Diabetes mellitus y riesgo cardiovascular. Recomendaciones del Grupo de Trabajo Diabetes Mellitas y Riesgo cardiovascular de la Sociedad Española de Diabetes 2006. Av. Diabetología [revista en Internet] 2006; 22(2): 11-6. Disponible en:

http://www.sediabetes.org/resources/revista/00010814archivoarticulo.pdf

15. Ministerio de Sanidad y Consumo. Estrategia en Diabetes del Sistema Nacional de Salud [Internet]. España: Ministerio de Sanidad y Consumo; 2007 [acceso 22 de febrero de 2009]. Disponible en:

http://www.msc.es/organizacion/sns/planCalidadSNS/pdf/excelencia/cuidadospaliativo s-diabetes/DIABETES/estrategia diabetes sistema nacional salud.pdf

16. World Health Organization. Diabetes mellitus. The cost of Diabetes. Disponible en:

http://www.who.int/mediacentre/factsheets/fs236

17. Tomás Abadal L, Varas Lorenzo C, Pérez I, Puig T, Balanguer Vintró I. Patrones de mortalidad en una cohorte de trabajadores seguida durante 28 años. Estudio Manresa. Rev Esp cardiol [revista en Internet] 2004 [acceso 22 de febrero de 2009]; 57:924-930. Disponible en:

http://www.revespcardiol.org/cgi-

bin/wdbcgi.exe/cardio/mrevista cardio.fulltext?pident $=13066452$

18. Sánchez- Chaparro MA, Román-García J, Calvo-Bonacho E, Gómez-Larios T, Fernández-Meseguer A, Sainz-Gutiérrez JC, et al. Prevalencia de factores de riesgo 
cardiovascular en una población laboral española. Rev Esp Cardiol [revista en Internet] 2006 [acceso 18 de febrero de 2009]; 59:421-430. Disponible en:

http://www.revespcardiol.org/cgi-

bin/wdbcgi.exe/cardio/mrevista cardio.resumen?pident=13087894

19. Castán Fernández FJ, Gutiérrez Bedmar M. Factores de riesgo cardiovascular y tipo de actividad en una población laboral. Rev INSHT [revista en Internet] 2004 [acceso 22 de febrero de 2009]; 29: 18-43. Disponible en:

http://www.mtas.es/insht/revista/A 29 ST.htm

20. Medrano MJ, Cerrato $E$, Boix R, Delgado-Rodríguez $M$. Factores de riesgo cardiovascular en la población española: metaánalisis de estudios transversales. Med Clin (Barc) [revista en Internet] 2005 [acceso 18 de febrero de 2009]; 124:606-612. Disponible en:

http://dialnet.unirioja.es/servlet/articulo?codigo=1154680

21. Banegas JR, Rodríguez-Artalejo F, Ruilope LM, Graciano A, Luque M, Cruz JJ, et al. Hypertension Magnitude and management in the elderly population of Spain. J Hypertens [revista en Internet] 2002 [acceso 14 de febrero de 2009]; 20:2157-2164.

Disponible en:

http://www.ncbi.nlm.nih.gov/pubmed/12409953

22. Kearney PM, Whelton M, Reynolds K, Muntner P, Whelton PK, He J. Global burden of hypertension: analysis of worldwide data. Lancet [revista en Internet] 2005 [acceso 14 de febrero de 2009]; 365:217-223. Disponible en:

http://www.ncbi.nlm.nih.gov/pubmed/15652604

23. Rigo Carratalá F, Guillen Frontera J, Llobera Cánaves J, Rodríguez Ruiz T, Borras Bosch I, Fuentespina Vidal E. Prevalencia de factores de riesgo cardiovascular en las Islas Baleares (estudio CORSAIB). Rev Esp Cardiol [revista en Internet] 2005 [acceso 22 de febrero de 2009]; 58: 11-19. Disponible en:

http://www.revespcardiol.org/cgi-

bin/wdbcgi.exe/cardio/mrevista cardio.fulltext?pident=13082539

24. Ministerio de Sanidad y Consumo. Encuesta Nacional de Salud 2006. Estilos de vida: Ejercicio físico en el tiempo libre población adulta [Internet]. España: INE (Instituto Nacional de Estadística); 2006 [acceso 20 de febrero de 2009]. Disponible en:

http://www.msc.es/estadEstudios/estadisticas/encuestaNacional/encuestaNac2006/Es tilosVidaPorcentaje.pdf

25. Vegazo García O, Civeira F, Banegas JR, Serrano Aisa PL, Jiménez FJ, Luengo E. Prevalencia de dislipemia en las consultas ambulatorias del Sistema Nacional de Salud. Estudio Hispalipid. Astra Zeneca [Internet]; 2005 [acceso 18 de febrero de 2009]. Disponible en:

http://www.saludvascular.es/article/517218.aspx

26. Grupo de Trabajo del Instituto Nacional de Seguridad e Higiene en el Trabajo. Factores de riesgo cardiovascular en la población laboral española. Rev INSHT [revista en Internet] 2000 [acceso 22 de febrero de 2009]; 5: 11-23. Disponible en: http://www.mtas.es/insht/revista/A 05 ST02.htm

27. Espina Álvarez C, Arcos González P, Canga Alonso A, Mallada Rivero L, Díaz Mora F. Riesgo de enfermedad coronaria en dos poblaciones laborales diferentes. Med Segur Trab. 2005; 51(200): 43-49.

ISSN 1695-6141

(C) COPYRIGHT Servicio de Publicaciones - Universidad de Murcia 